hyperaemic coronary flow reserve. Thus, we hypothesised that patients in AF would achieve splenic switch-off less frequently than those in sinus rhythm (SR).

Methods 1100 adenosine stress CMR studies were retrospectively identified from a prospectively maintained clinical CMR database over a 5-year period (2016-2021). 71 patients in AF on ECG at the time of CMR were identified. The SR cohort was drawn by propensity score matching to the AF group for age, sex, and body mass index. The adenosine dose administered, symptoms, and heart-rate change observed during infusion and scan result were recorded. Splenic switch-off was evaluated subjectively through visual assessment and semiquantitatively via changes in splenic and myocardial signal intensity from rest to stress. Multivariate logistic regression analysis assessed for predictors of splenic switch-off.

Results 70 AF patients were matched to 70 in SR (mean age: $72 \pm 8$ years vs $72 \pm 9$ years, $p=0.96 ; \%$ male: $86 \%$ vs $84 \%$, $\mathrm{p}=0.81$ ). Subjective splenic switch-off occurred significantly less frequently in AF than in SR (34/70 [49\%] vs 53/70 [76\%], $\mathrm{p}=0.003)$. Objective assessment supported this, with a smaller splenic signal intensity difference between stress and rest in AF vs SR (median splenic stress:rest peak signal intensity ratio 0.92 [IQR, 0.61-1.11] vs 0.56 [IQR, 0.45-0.75], $\mathrm{p}<0.001)$. A heart-rate increase over $10 \mathrm{bpm}$ in response to adenosine was a significant predictor of visual splenic switchoff in SR patients but not in AF patients. Fewer AF patients had inducible ischaemia than SR patients (9/70 [13\%] vs 17/ 69 [25\%], $\mathrm{p}=0.058)$. However, this difference was not driven by inducible ischaemia rates between $\mathrm{AF}$ and SR patients who did not achieve splenic switch-off (6/36 [17\%] vs 4/17 [24\%], $\mathrm{p}=0.403$ ).

Conclusions In this retrospective propensity matched study, splenic switch-off occurred significantly less frequently in patients with AF. This may risk the under-diagnosis of inducible ischaemia, but requires further assessment.

\section{RATIONALE AND DESIGN OF THE MEDICAL RESEARCH COUNCIL PRECISION MEDICINE WITH ZIBOTENTAN IN MICROVASCULAR ANGINA (PRIZE) TRIAL MRI SUB-STUDY}

Andrew Morrow, ${ }^{1,2}$ Thomas J Ford, ${ }^{1}$ Robert Sykes, ${ }^{1}$ Kenneth Mangion, ${ }^{4}$ Tushar Kotecha, ${ }^{4}$ Roby Rakhit, ${ }^{5}$ Gavin Galasko, ${ }^{6}$ Stephen Hoole, ${ }^{7}$ Anthony Davenport, ${ }^{7}$ George Abraham, ${ }^{8}$ Rajesh Kharbanda, ${ }^{8}$ Vanessa Ferreira, ${ }^{9}$ Amedeo Chiribiri, ${ }^{10}$ Divaka Perera, ${ }^{10}$ Haseeb Raman, ${ }^{11}$ Jayanth R Arnold, ${ }^{12}$ John P Greenwood, ${ }^{13}$ Michael Fisher, ${ }^{3}$ Dirk Husmeier, ${ }^{3}$ Nicholas A Hill, ${ }^{3}$ Xiaoyu Luo, ${ }^{14}$ Nicola Williams, ${ }^{14}$ Laura Miller, ${ }^{1}$ Jill Dempster, ${ }^{1}$ Peter W Macfarlane, ${ }^{1}$ Paul Welsh, ${ }^{1}$ Naveed Sattar, ${ }^{15}$ Andrew Whittaker, ${ }^{16}$ Alex McConnachie, 'Sandosh Padmanabhan, ${ }^{1}$ Colin Berry. ${ }^{1}$ British Heart Foundation Glasgow Cardiovascular Research Centre, Institute of Cardiovascular and Medical Sciences, University of Glasgow, Glasgow, UK; ${ }^{2}$ University of New South Wales, Sydney, Australia; ${ }^{3}$ School of Mathematics and Statistics, University of Glasgow, Glasgow, UK; ${ }^{4}$ Royal Free Hospital, Royal Free London NHS Foundation Trust London, UK; ${ }^{5}$ Lancashire Cardiac Centre, Blackpool Teaching Hospitals NHS Foundation Trust, Blackpool, UK; ${ }^{6}$ Department of Interventional Cardiology, Royal Papworth Hospital, Cambridge, UK; ${ }^{7}$ Clinical Pharmacology, University of Cambridge, Cambridge, UK; ${ }^{8}$ Raddliffe Department of Cardiovascular Medicine, University of Oxford, Oxford, UK; ${ }^{9}$ Division of Imaging Sciences, Guy's and St Thomas' Hospital NHS Foundation Trust, London, UK; ${ }^{10}$ School of Cardiovascular Medicine and Sciences, King's College London, London, UK; ${ }^{11}$ Department of Cardiovascular Sciences, Glenfield Hospital, Leicester, UK; ${ }^{12}$ Leeds University and Leeds Teaching Hospitals NHS Trust, Leeds, UK; ${ }^{13}$ Liverpool University and Liverpool University Hospitals NHS Foundation Trust, Liverpool, UK; ${ }^{14}$ Department of Clinical Genetics, Queen Elizabeth University Hospital, Glasgow, UK; ${ }^{15}$ Emerging Innovations Unit, Discovery Sciences, RandD, AstraZeneca, Cambridge, UK; ${ }^{16}$ Robertson Centre for Biostatistics, Institute of Health and Wellbeing, University of Glasgow, Glasgow, UK

10.1136/heartjnl-2021-BSCMR.3
Introduction Microvascular angina is caused by cardiac small vessel disease and dysregulation of the endothelin system is implicated. The chronic elevation of circulating ET-1 in microvascular angina may be influenced by genetic factors. The minor $G$ allele of the non-coding single nucleotide polymorphism (SNP) rs9349379 enhances expression of the endothelin 1 gene in human vascular cells, increasing circulating concentrations of ET-1. The prevalence of this allele is higher in patients with microvascular angina. Zibotentan is a potent, selective inhibitor of the ETA receptor. We have identified zibotentan as a potential disease-modifying therapy for patients with microvascular angina.

The Precision medicine with Zibotentan in microvascular angina (PRIZE) trial is a prospective, randomized, doubleblind, placebo-controlled, sequential cross-over trial. We will assess the efficacy and safety of adjunctive treatment with oral zibotentan (10 $\mathrm{mg}$ daily) in patients with microvascular angina and assess whether rs9349379 (minor G allele; population prevalence $\sim 36 \%$ ) acts as a theragnostic biomarker of the response to treatment with zibotentan. The participants will receive a single-blind placebo run-in followed by treatment with either $10 \mathrm{mg}$ of zibotentan daily for 12 weeks then placebo for 12 weeks, or vice versa, in random order.

After randomisation in PRIZE, subjects will be invited to participate in the cardiac MRI sub-study. Myocardial perfusion is generally impaired in patients with microvascular angina. The rationale for undertaking this MRI sub-study is to determine whether, compared with placebo, treatment with zibotentan improves myocardial blood flow.

Methods Patients will undergo multiparametric CMR at three points (baseline prior to therapy and after each 12 week treatment phase). At each scan they will have assessments of myocardial blood flow (both rest and stress using the Kellman quantitative perfusion method), left ventricular function and mass, aortic stiffness assessment and tissue characterisation (T1 mapping (MOLLI and ShMOLLI), T2 mapping and late gadolinium enhancement imaging).

Conclusion PRIZE invokes precision medicine in microvascular angina. Should our hypotheses be confirmed, this developmental trial will inform the rationale and design for undertaking a larger multicentre trial. The MRI sub-study contributes to this study by providing vital mechanistic and safety information.

\section{\begin{tabular}{l|l}
4 & INTER-MODALITY AGREEMENT AND TEST-RETEST
\end{tabular} REPRODUCIBILITY OF CMR AND ECHOCARDIOGRAPHY FOR ASSESSING MYOCARDIAL DEFORMATION IN TYPE 2 DIABETES MELLITUS}

Aseel Alfuhied, Gaurav S Gulsin, Emer M Brady, Jian L Yeo, Kelly Parke, Anna-Marie Marsh, Jayanth Ranjit Arnold, Gerry P McCann, Anvesha Singh. Department of Cardiovascular Sciences, University of Leicester and Cardiovascular Theme, National Institute for Health Research (NIHR) Leicester Biomedical Research Centre, Glenfield Hospital, Leicester, UK

\subsection{6/heartjnl-2021-BSCMR.4}

Background Inter-modality agreement allows interchangeable use of imaging modalities that is crucial for clinical decisionmaking, while the reproducibility of a technique is fundamental for monitoring disease progression or response to treatment. We aimed to investigate the test-retest reproducibility and inter-modality agreement of transthoracic echocardiography (TTE) and cardiac magnetic resonance (CMR) imaging in assessing left ventricular (LV) and left atrial (LA) myocardial deformation in people with type 2 diabetes mellitus (T2D). 
Abstract 4 Table 1 Test-retest reproducibility of myocardial deformation in TTE and CMR

\begin{tabular}{|c|c|c|c|c|c|c|}
\hline Parameter & $\begin{array}{l}\text { Scan } 1 \text { (Mean } \\
\pm \text { SD) }\end{array}$ & $\begin{array}{l}\text { Scan } 2 \text { (Mean } \\
\pm \text { SD) }\end{array}$ & $\begin{array}{l}\text { Bias (Limits of } \\
\text { agreement) }\end{array}$ & ICC & $\begin{array}{l}\text { ICC_p- } \\
\text { value }\end{array}$ & $\begin{array}{l}\text { CoV } \\
(\%)\end{array}$ \\
\hline \multicolumn{7}{|l|}{ TTE } \\
\hline LV_GLS (\%) & $-17.2 \pm 2.4$ & $-16.8 \pm 1.6$ & $-0.37(-5.44,4.70)$ & 0.36 & 0.272 & 15.25 \\
\hline LV_MCS & $-25.4 \pm 4.0$ & $-23.9 \pm 2.8$ & $-1.54(-11.9,8.86)$ & 0.4 & 0.686 & 21.53 \\
\hline \multicolumn{7}{|l|}{ (\%) } \\
\hline LAS_r (\%) & $33.8 \pm 3.7$ & $31.4 \pm 6.8$ & $2.45(-10.9,15.8)$ & 0.35 & 0.26 & 20.9 \\
\hline LAS_cd (\%) & $16.7 \pm 3.4$ & $15.0 \pm 4.2$ & $1.64(-4.11,7.38)$ & 0.8 & $0.008^{*}$ & 18.5 \\
\hline LAS_bp (\%) & $17.1 \pm 3.2$ & $16.3 \pm 4.4$ & $0.81(-9.17,10.8)$ & 0.23 & 0.362 & 30.4 \\
\hline \multicolumn{7}{|l|}{ CMR } \\
\hline LV_GLS (\%) & $-17.4 \pm 2.2$ & $-17.0 \pm 2.5$ & $-0.37(-5.11,4.36)$ & 0.65 & 0.078 & 14.1 \\
\hline LV_MCS & $-19.9 \pm 2.0$ & $-19.2 \pm 3.6$ & $-0.66(-7.18,5.86)$ & 0.53 & 0.146 & 17 \\
\hline \multicolumn{7}{|l|}{ (\%) } \\
\hline LAS_r (\%) & $29.2 \pm 6.5$ & $27.9 \pm 7.8$ & $1.26(-9.74,12.3)$ & 0.83 & $0.009^{*}$ & 19.6 \\
\hline LAS_cd (\%) & $15.1 \pm 6.0$ & $12.6 \pm 5.3$ & $2.57(-7.30,12.4)$ & 0.73 & $0.024^{*}$ & 36.3 \\
\hline LAS_bp (\%) & $14.0 \pm 5.9$ & $15.4 \pm 6.0$ & $-1.31(-11.4,8.73)$ & 0.78 & $0.020^{*}$ & 34.9 \\
\hline
\end{tabular}

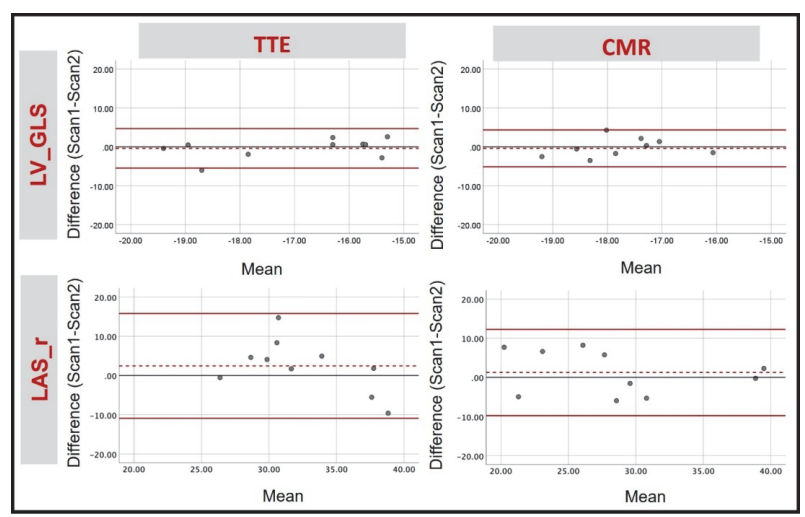

Abstract 4 Figure 1 Bland-Altman plot for test-retest reproducibility of LV global longitudinal strain and LA reservoir strain in TTE and CMR

Methods Participants with T2D and no cardiovascular disease

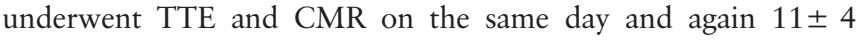
days later. Images were analysed using TomTec-ARENA (v2.4, 2D-CPA) for TTE, where Medis Suite (v3.1, medical imaging system) was used for CMR images. LV global longitudinal strain (GLS) was calculated using an average of 2-, 3- and 4chamber values and LV mid-circumferential strain (LV_MCS) was calculated from the mid short-axis cine at papillary muscles level. In addition, LA strain (LAS), corresponding to LA reservoir, conduit, and booster pump (contraction function), was calculated using the average of 4- and 2-chamber values. The exact same analysis technique was used for both imaging modalities, and blinded analysis was performed by the same observer.

Results 10 participants with T2D (mean age 65.6 77.3 years, $50 \%$ male) were studied. The LV_MCS and reservoir LAS were significantly lower on CMR compared to TTE, though there was no difference in GLS, with narrow limits of agreement between CMR and TTE values. Of all strain parameters, GLS had the best test-retest reproducibility, with almost identical bias and limits of agreement on Bland-Altman analysis and similar CoV ( 15\%). The test-retest reproducibility for LV_MCS and reservoir LAS were better on CMR than TTE (table 1 \& figure 1). TTE was more reproducible for conduit LAS (CoV 18.5\%, ICC $=0.80)$.

Conclusion Both CMR and TTE gave similar values for GLS, with good inter-modality agreement and equally good testretest reproducibility, suggesting the potential use of both modalities for monitoring GLS in disease and in response to treatment. The test-retest reproducibility of LV_MCS and reservoir LAS was better on CMR, whilst TTE was better for assessing conduit LAS.

\section{\begin{tabular}{|l|l}
\hline 5 & ABSTRACT WITHDRAWN
\end{tabular}}

